\title{
ATUAÇÃO DA ENFERMAGEM MEDIANTE A PREVENÇÃO E DETECÇÃO PRECOCE DE SEPSE NA UNIDADE DE TERAPIA INTENSIVA: UMA REVISÃO
}

\section{NURSING PERFORMANCE THROUGH THE PREVENTION AND EARLY DETECTION OF SEPSIS IN THE INTENSIVE CARE UNIT: A REVIEW}

\section{DESEMPEÑO DE ENFERMERÍA MEDIANTE LA PREVENCIÓN Y DETECCIÓN TEMPRANA DE SEPSIS EN LA UNIDAD DE CUIDADOS INTENSIVOS: UNA REVISIÓN}

\section{Maressa Samai Pinheiro Silva Smith ${ }^{1}(\mathbb{D}$ Averlândio Wallysson Soares da Costa²}

\author{
${ }^{1}$ Graduada em Enfermagem pela Universidade Potiguar (UNP), Natal, RN, Brasil; ${ }^{2}$ Professor da Escola de Saúde da Universidade \\ Potiguar (UNP). Mestre em Saúde Coletiva, Natal, RN, Brasil. \\ *Autores correspondentes: maressa-samai@,hotmail.com; averlandio.costa@unp.br
}

Recebido: 13/09/2021 | Aprovado: 06/11/2021 | Publicado: 16/11/2021

Resumo: $\mathrm{O}$ objetivo deste estudo consiste em identificar quais os impactos que a atuação da enfermagem proporciona perante a prevenção e detecção precoce de sepse na assistência ao paciente que se encontra na Unidade de Terapia Intensiva (UTI). Trata-se de uma revisão integrativa de literatura, a busca foi realizada nas bases de dados eletrônicas: Scientific Eletronic Library Online (SciELO), Biblioteca Virtual em Saúde do Ministério da Saúde (BVSMS) e Literatura Latino-Americana e do Caribe em Ciências da Saúde (LILACS). Utilizou-se os Descritores em Ciência da Saúde (DeCS): "enfermagem", "Unidade de Terapia Intensiva", "cuidados de enfermagem", "sepse". Após análise dos estudos, 21 artigos foram selecionados para constituir a amostra. A equipe de enfermagem intensivista é imprescindível na prevenção, tratamento e diagnóstico da sepse. Ademais, é percebido que a implementação de ações de cunho educativo aos profissionais envolvidos impacta diretamente na melhoria do nível de conhecimento, gestão e prática assistencial a esses pacientes. Assim, saliente-se que a compreensão sobre a doença influencia em um monitoramento eficaz, contribuindo para identificar os sinais iniciais da sepse, um fim de que seja proporcionado uma intervenção estratégica no menor tempo possível na unidade de terapia intensiva.

Palavras-chave: Enfermagem. Unidade de Terapia Intensiva. Cuidados de enfermagem. Sepse.

Abstract: The aim of this study is to identify the impacts that nursing activies provide towards the prevention and early detection of sepsis in patient care in the Intensive Care Unit (ICU). This is an integrative literature review, the seach was carried out in eletrnic databases: Scientific Eletronic Library Online (SciELO), Ministry of Health Virtual Health Library (BVSMS) and Latin American an Caribbean Literature in Sciences of the Heath (LILACS). The Health Science Descriptors (DeCS) were used: "nursing”, " Intensive Care Unit", "nursing care", "sepsis". After analyzing the studies, 21 articles were selected to constitute the sample. Furthermore, it is perceived that the implementation of educational actions for the professionals involved directly impacting the improvement of the level of knowledge, management and care practice for these patients. Thus, it is noteworthy that the inderstanding of the disease influences effective monitoring, helping to identify the initial signs of sepsis, in order to provide a strategic intervention in the shortest time possible in the intensive care.

Keywords: Nursing. Intensive Care Unit. Nursing care. Sepsis.

Resumen: El objetivo de este estudio es identificar los impactos que las actividades de enfermería brindan en la prevención y detección precoz de la sepsis en la atención al paciente en la Unidad de Cuidados Intensivos (UCI). 
Se trata de una revisión integradora de la literatura, la búsqueda se realizó en bases de datos eletrónicas: Biblioteca Electrónica Científica en Línea (SciELO), Biblioteca Virtual en Salud del Ministerio de Salud (BVSMS) y Literatura Latinoamericana y del Caribe en Ciencias de la Salud (LILACS). Se utilizaron los Descriptores de la Salud (DeCS): "enfermería", "Unidad de Cuidados Intensivos", "cuidados de enfermería", "sepsis". Tras analizar los estudios, se seleccionaron 21 artículos para constituir la muestra. Además, se percibe que la implementación de acciones formativas para los profesionales involucrados impacta directamente en la mejora del nivel de conocimiento, gestión y práctica asistencial de estos pacientes. Así, es de destacar que el conocimiento de la enfermedad influye en el seguimiento efectivo, ayudando a identificar los signos iniciales de sepsis, com en fin de brindar una intervensión estratégica en el menor tiempo posible en la unidad de cuidados intensivos.

Palabras clave: Enfermería. Unidad de Cuidados Intensivos. Cuidado de enfermera. Septicemia.

\section{INTRODUÇÃO}

Considerada uma disfunção orgânica, a sepse pode ser ocasionada por uma resposta imune desregulada à uma infeção, manifestando-se em múltiplos estágios clínicos oriundos de um mesmo processo fisiológico. Os principais focos infecciosos iniciais são o trato respiratório, urinário e gastrointestinal, os quais estão relacionados a agentes etiológicos como fungos, bactérias e determinados vírus, como é o caso do Sars Cov-2, que pode desencadear processo de sepse em pacientes com COVID-19 em estágio grave (Pedrosa, Oliveira \& Machado, 2018; Ramalho Neto et al., 2019; Souza et al., 2020).

No cenário epidemiológico, a incidência dessa patologia vem se exacerbando de modo importante nas últimas décadas. Estimativas demonstram que ocorram aproximadamente 5 milhões de mortes por sepse no mundo por ano, com incidência em torno de 31 milhões de casos anualmente. Nesse contexto, é importante salientar que a sepse é capaz de apresentar uma evolução potencialmente fatal, e o âmbito hospitalar é o principal ambiente para propiciar o seu desenvolvimento, atingindo comumente pacientes críticos, consistindo em uma afecção relevante na unidade de terapia intensiva (UTI). Uma vez que, a probabilidade de novas infecções nesse grupo é de três a cinco vezes maior do que indivíduos nunca acometidos por sepse (Zonta et al., 2018; Taniguchi et al., 2019).

Essa complicação é considerada uma das principais causas de óbitos na terapia intensiva, demandando uma assistência meticulosa devido à complexidade do atendimento, que em sua maioria é classificada como urgência. Nessa conjuntura, obtendo conhecimento sobre a criticidade da doença, é notório que a detecção precoce e uma prevenção com afinco são essenciais para atenuar as chances de morbimortalidade do paciente. Além de consequentemente reduzir os altos custos em saúde para as instituições (Moura et al., 2019; Branco et al., 2020).

Diante disso, os cuidados da equipe de enfermagem podem impactar positivamente na saúde do indivíduo acometido por essa disfunção orgânica. Uma vez que os profissionais estejam qualificados para tal, seus cuidados prestados de maneira adequada, contínua e sistematizada visam a prevenção, reconhecimento da situação e de fatores de risco para seu desenvolvimento nos pacientes. Assim como, a detecção precoce de 
complicações e intervenção no menor tempo hábil e de forma eficiente proporcionam zelo e a recuperação do paciente (Pedrosa, Oliveira \& Machado, 2018; Branco et al., 2020).

Nesta perspectiva, perante a importância da equipe de enfermagem no atendimento ao séptico, o estudo apresenta como objetivo, analisar o impacto da atuação da Enfermagem na prevenção e detecção precoce de sepse em pacientes que se encontram na Unidade de Terapia Intensiva (UTI).

\section{MATERIAL E MÉTODOS}

Este estudo trata-se de uma revisão de literatura integrativa, a qual possibilita que a pesquisa científica seja analisada de forma ampla favorecendo a dissipação do conhecimento produzido. De acordo com Gonçalves (2019), é um método de pesquisa sequenciada por cinco etapas para sua construção: elaboração da questão norteadora; busca na literatura; coleta de dados; análise crítica dos estudos incluídos; interpretação e síntese dos resultados; e apresentação dos resultados. Para a elaboração deste estudo foi adotada a seguinte questão norteadora: "Quais os impactos da assistência de enfermagem para prevenção e detecção precoce da sepse em Unidade de Terapia Intensiva".

Visando a resolutividade da questão supracitada, foram realizadas pesquisas de estudos disponíveis nas bases de dados eletrônicas: Scientific Eletronic Library Online (SciELO), Biblioteca Virtual em Saúde do Ministério da Saúde (BVSMS) e Literatura Latino-Americana e do Caribe em Ciências da Saúde (LILACS). Para a seleção da pesquisa foram utilizados descritores padronizados pelos Descritores em Ciência da Saúde (DeCS): “enfermagem”, “Unidade de Terapia Intensiva”, “cuidados de enfermagem”, "sepse”, com operador boleano AND.

Estabelecendo os critérios de inclusão, foram inseridos artigos disponibilizados de forma gratuita e em sua integralidade eletronicamente, que abordassem a temática empregada entre os anos de 2016 a agosto de 2021, em língua portuguesa, inglesa e espanhola. Foi empregado como critério de exclusão: dissertações, teses, produções duplicadas, relato de casos informais, artigos incompletos ou não disponibilizados gratuitamente e que não possuíssem relação com a temática ou que não respondessem à questão norteadora abordada.

As informações obtidas para a construção deste estudo, foi realizada por meio de consulta online entre os meses de julho e agosto de 2021. O processo de seleção, elegibilidade e inclusão dos estudos analisados, estão descritos na Figura 1.

Figura 1 - Fluxograma da seleção de estudos incluídos na revisão integrativa. Natal, Rio Grande do Norte. Brasil, 2021. 


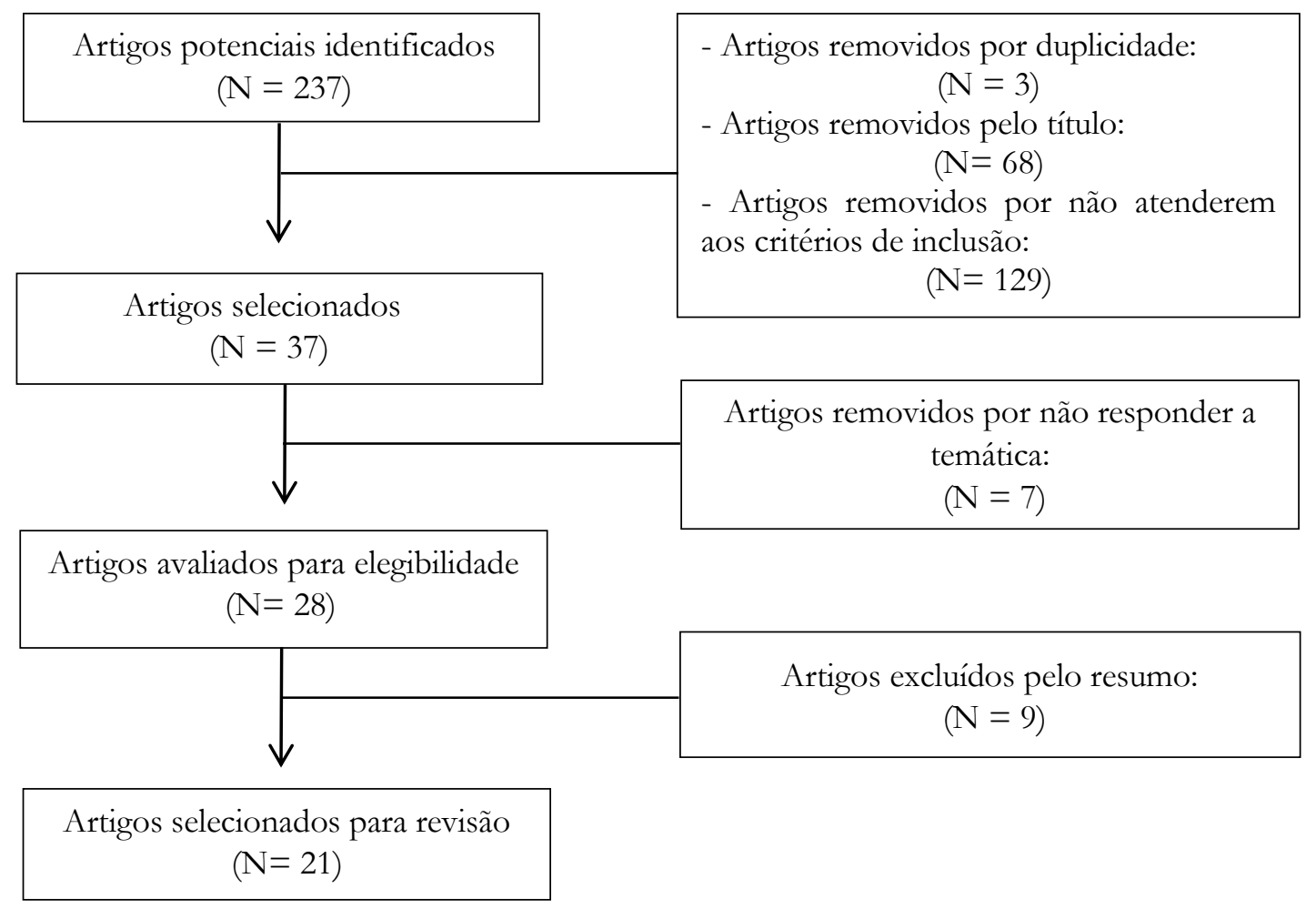

Fonte: Elaboração dos autores, 2021.

\section{RESULTADOS}

Em consonância com os critérios de elegibilidade da metodologia, foram incluídos "21" artigos. A sumarização desses estudos que constituem a amostra da presente revisão, estão dispostas no quadro 1.

Quadro 1 - Amostra dos resultados dos artigos selecionados para pesquisa, após aplicados os critérios de inclusão e exclusão. Natal, Rio Grande do Norte. Brasil, 2021.

\begin{tabular}{|c|c|c|c|}
\hline $\begin{array}{l}\text { Autor } \\
\text { (Ano) }\end{array}$ & Título & Resultados & Tipo de estudo \\
\hline $\begin{array}{c}\text { Barros, Maia \& } \\
\text { Monteiro } \\
(2016)\end{array}$ & $\begin{array}{c}\text { Fatores de risco } \\
\text { associados ao } \\
\text { agravamento de sepse } \\
\text { em pacientes em } \\
\text { Unidade de Terapia } \\
\text { Intensiva }\end{array}$ & $\begin{array}{l}\text { Foram estudados } 212 \text { pacientes na } \\
\text { UTI, dos quais } 181 \text { apresentavam } \\
\text { sepse. Os fatores de risco associados } \\
\text { ao agravamento da sepse foram: idade } \\
\text { superior que } 65 \text { anos, maior tempo } \\
\text { médio de internação na UTI, elevada } \\
\text { frequência de comorbidades e a } \\
\text { utilização de procedimentos invasivos. }\end{array}$ & $\begin{array}{c}\text { Estudo } \\
\text { observacional } \\
\text { descritivo }\end{array}$ \\
\hline $\begin{array}{l}\text { Fernandes et al. } \\
\text { (2016) }\end{array}$ & $\begin{array}{l}\text { Atuação da enfermagem } \\
\text { na detecção precoce e } \\
\text { tratamento da sepse na } \\
\text { terapia intensiva }\end{array}$ & $\begin{array}{l}\text { Os cuidados de enfermagem diante a } \\
\text { sepse conforme as necessidades é } \\
\text { através da consulta qualificável de } \\
\text { enfermagem, ao observar anamnese, } \\
\text { histórico clínico e da terapêutica } \\
\text { fomentada pela equipe } \\
\text { multidisciplinar, considerando } \\
\text { critérios sistêmicos que possam surgir. }\end{array}$ & Revisão integrativa \\
\hline Torsvik et al. & $\begin{array}{l}\text { Identificação precoce de } \\
\text { sepse em internação }\end{array}$ & $\begin{array}{l}\text { Um total de } 478 \text { pacientes foram } \\
\text { admitidos no período pré-intervenção }\end{array}$ & $\begin{array}{c}\text { Estudo de } \\
\text { intervenção }\end{array}$ \\
\hline
\end{tabular}




\begin{tabular}{|c|c|c|c|}
\hline$(2016)$ & $\begin{array}{c}\text { hospitalar por } \\
\text { enfermeiros de } \\
\text { enfermaria aumenta } 30 \\
\text { dias de sobrevida }\end{array}$ & $\begin{array}{l}\text { e } 422 \text { no período pós intervenção. O } \\
\text { grupo pós-intervenção foi melhor } \\
\text { observado e apresentou maiores } \\
\text { chances de sobreviver } 30 \text { dias, menor } \\
\text { probabilidade de desenvolver falência } \\
\text { de órgãos graves menor que o grupo } \\
\text { pré-intervenção. }\end{array}$ & \\
\hline $\begin{array}{l}\text { Kleinpell } \\
(2017)\end{array}$ & $\begin{array}{l}\text { Promover a identificação } \\
\text { precoce de sepse em } \\
\text { pacientes hospitalizados } \\
\text { com protocolos } \\
\text { conduzidos por } \\
\text { enfermeiras }\end{array}$ & $\begin{array}{l}\text { O papel dos enfermeiros na melhoria } \\
\text { da qualidade do cuidado com a sepse é } \\
\text { significativo. Como os enfermeiros } \\
\text { passam a maior parte do tempo com } \\
\text { os pacientes, seu papel no } \\
\text { reconhecimento e tratamento de } \\
\text { pacientes com sepse é fundamental } \\
\text { para melhorar os desfechos } \\
\text { relacionados à sepse. }\end{array}$ & $\begin{array}{c}\text { Estudo } \\
\text { retrospectivo }\end{array}$ \\
\hline $\begin{array}{l}\text { Moura et al. } \\
\text { (2017) }\end{array}$ & $\begin{array}{l}\text { Diagnóstico de sepse em } \\
\text { pacientes após } \\
\text { internação em unidade } \\
\text { de terapia intensiva }\end{array}$ & $\begin{array}{l}\text { Maior número de internação foi do } \\
\text { sexo masculino, com idade entre } 51 \text { e } \\
70 \text { anos. As comorbidades mais } \\
\text { encontradas foram hipertensão arterial } \\
\text { sistêmica, Diabetes Mellitus, etilismo, } \\
\text { tabagismo e cardiopatia. }\end{array}$ & $\begin{array}{c}\text { Estudo } \\
\text { retrospectivo } \\
\text { propriamente dito, } \\
\text { de campo, } \\
\text { descritivo de } \\
\text { abordagem } \\
\text { quantitativa }\end{array}$ \\
\hline $\begin{array}{l}\text { Garrido et al. } \\
\text { (2018) }\end{array}$ & $\begin{array}{l}\text { Ações do enfermeiro na } \\
\text { identificação precoce de } \\
\text { alterações sistêmicas } \\
\text { causadas pela sepse } \\
\text { grave }\end{array}$ & $\begin{array}{c}\text { Apenas } 36 \% \text { dos enfermeiros } \\
\text { possuem especialização em UTI } \\
\text { adulto; verificou-se que os } \\
\text { profissionais identificam parcialmente } \\
\text { os sinais e sintomas apresentados pelo } \\
\text { paciente séptico. }\end{array}$ & Estudo descritivo \\
\hline $\begin{array}{c}\text { Pedrosa, Oliveira \& } \\
\text { Machado } \\
\text { (2018) }\end{array}$ & $\begin{array}{l}\text { Validação de protocolo } \\
\text { assistencial ao paciente } \\
\text { séptico na Unidade de } \\
\text { Terapia Intensiva }\end{array}$ & $\begin{array}{l}\text { A validação de conteúdo referente à } \\
\text { assistência do enfermeiro ao paciente } \\
\text { séptico em terapia intensiva } \\
\text { inicialmente foi composto por dezoito } \\
\text { itens analisados pelos } \\
\text { avaliadores/juízes. Deste, por meio do } \\
\text { Índice de Validade de Conteúdo } \\
\text { (IVC), identificou-se treze itens com } \\
\text { forte evidência de validação. }\end{array}$ & $\begin{array}{c}\text { Estudo de validação } \\
\text { metodológica de } \\
\text { instrumento }\end{array}$ \\
\hline $\begin{array}{l}\text { Scheidt et al. } \\
\qquad(2018)\end{array}$ & $\begin{array}{l}\text { Implantação do } \\
\text { protocolo de Manejo de } \\
\text { sepse o pronto } \\
\text { atendimento do hospital } \\
\text { universitário regional } \\
\text { dos campos gerais. }\end{array}$ & $\begin{array}{c}\text { Os pacientes submetidos ao } \\
\text { protocolo tinham idade média de } 66 \\
\text { anos, a maioria eram }(56 \%) \text { mulheres, } \\
\text { apresentaram hipotensão }(96 \%) \text {, } \\
\text { elevação da creatinina }(76 \%) \text { e } \\
\text { leucocitose }(70 \%) .\end{array}$ & $\begin{array}{l}\text { Estudo descritivo, } \\
\text { do tipo transversal }\end{array}$ \\
\hline $\begin{array}{c}\text { Silva \& Souza } \\
\text { (2018) }\end{array}$ & $\begin{array}{l}\text { Sepse: importância da } \\
\text { identificação precoce } \\
\text { pela enfermagem }\end{array}$ & $\begin{array}{c}\text { Pelo fato de esses profissionais } \\
\text { permanecerem à beira do leito, eles } \\
\text { devem estar aptos a identificar os } \\
\text { sinais e sintomas da sepse e planejar a } \\
\text { assistência de enfermagem, de acordo } \\
\text { com as necessidades de cuidado ao } \\
\text { paciente. }\end{array}$ & Revisão integrativa \\
\hline $\begin{array}{l}\text { Zonta et al. } \\
\text { (2018) }\end{array}$ & $\begin{array}{c}\text { Características } \\
\text { epidemiológicas e } \\
\text { clínicas da sepse em um } \\
\text { hospital público do }\end{array}$ & $\begin{array}{l}\text { Houve predominância de pacientes do } \\
\text { sexo masculino, faixa etária maior de } \\
70 \text { anos e cor branca. O tempo de } \\
\text { internação foi de até uma semana. }\end{array}$ & $\begin{array}{l}\text { Pesquisa de campo } \\
\text { exploratória, } \\
\text { documental, } \\
\text { retrospectiva, com }\end{array}$ \\
\hline
\end{tabular}




\begin{tabular}{|c|c|c|c|}
\hline & Paraná & $\begin{array}{l}\text { Prevaleceram pacientes clínicos, com } \\
\text { infecção nosocomial encontrada em } \\
\text { dos casos. As principais fontes de } \\
\text { infecção foram pulmão e ferida } \\
\text { cirúrgica. }\end{array}$ & $\begin{array}{l}\text { abordagem } \\
\text { quantitativa }\end{array}$ \\
\hline $\begin{array}{l}\text { Goulart et al. } \\
\text { (2019) }\end{array}$ & $\begin{array}{l}\text { Os enfermeiros estão } \\
\text { atualizados sobre o } \\
\text { manejo adequado de } \\
\text { pacientes com sepse? }\end{array}$ & $\begin{array}{c}\text { Apenas } 16,6 \% \text { dos profissionais } \\
\text { receberam treinamento em serviço } \\
\text { sobre o tema. Não houve implantação } \\
\text { de protocolos de sepse na instituição, } \\
\text { embora } 96,6 \% \text { dos participantes } \\
\text { considerem sua implementação } \\
\text { necessária. }\end{array}$ & Estudo descritivo \\
\hline $\begin{array}{l}\text { Moura et al. } \\
\text { (2019) }\end{array}$ & $\begin{array}{c}\text { Produção Científica } \\
\text { Sobre Sepse, Terapia } \\
\text { Intensiva e } \\
\text { Enfermagem: Análise } \\
\text { Bibliométrica do } \\
\text { Período de 2003-2018 }\end{array}$ & $\begin{array}{c}\text { Na busca inicial pela palavra } \\
\text { "Sepse” foram encontrados } 26.923 \\
\text { resultados, ao buscar "Sepse And UTI } \\
2.921 \text { resultados, e ao refinar para } \\
\text { "Sepse and UTI and enfermagem" o } \\
\text { resultado abrangente encontrado } \\
\text { Foi de } 82 \text { estudos. Na busca específica } \\
\text { para "Sepse, UTI e } \\
\text { Enfermagem" a ênfase clínica foi dada } \\
\text { à etiologia( }(37 \%) ; \\
\text { Prognóstico }(18 \%) \text { e terapia (15\%). O } \\
\text { tipo de documento } \\
\text { mais frequentemente publicado, } \\
\text { representando } 82 \text { dos } 53 \text { resultados. }\end{array}$ & $\begin{array}{c}\text { Pesquisa } \\
\text { bibliométrica, de } \\
\text { abordagem } \\
\text { quantitativa }\end{array}$ \\
\hline $\begin{array}{l}\text { Ramalho Neto et al. } \\
\qquad(2019)\end{array}$ & $\begin{array}{c}\text { Paciente grave com } \\
\text { sepse: concepções e } \\
\text { atitudes de enfermeiros } \\
\text { intensivistas }\end{array}$ & $\begin{array}{l}\text { Da análise qualitativa emergiram duas } \\
\text { categorias: compreensão sobre a sepse } \\
\text { e cuidados intensivos ao paciente com } \\
\text { sepse. Os depoimentos dos } \\
\text { enfermeiros intensivistas revelaram } \\
\text { adequado conhecimento sobre a } \\
\text { sepse, com ações embasadas na } \\
\text { Campanha Sobrevivendo à Sepse e na } \\
\text { própria experiência clínica. }\end{array}$ & $\begin{array}{c}\text { Estudo exploratório } \\
\text { com abordagem } \\
\text { qualitativa }\end{array}$ \\
\hline $\begin{array}{l}\text { Oliveira et al. } \\
\text { (2019) }\end{array}$ & $\begin{array}{c}\text { O enfermeiro na } \\
\text { detecção dos sinais e } \\
\text { sintomas que antecedem } \\
\text { sepse em pacientes na } \\
\text { enfermaria }\end{array}$ & $\begin{array}{c}\text { Evidenciou-se que possuem } \\
\text { entendimento sobre o conceito de } \\
\text { sepse, entretanto apresentaram } \\
\text { dificuldades em correlacionar alguns } \\
\text { dos sinais e sintomas dos tipos de } \\
\text { sepse. }\end{array}$ & Estudo descritivo \\
\hline $\begin{array}{l}\text { Taniguchi et al. } \\
\text { (2019) }\end{array}$ & $\begin{array}{l}\text { Disponibilidade de } \\
\text { recursos para tratamento } \\
\text { da sepse no Brasil: uma } \\
\text { amostra aleatória de } \\
\text { instituições brasileiras }\end{array}$ & $\begin{array}{c}\text { As instituições com alta } \\
\text { disponibilidade de recursos (158 } \\
\text { unidades; } 57 \% \text { ) eram, em geral, } \\
\text { maiores e atendiam principalmente } \\
\text { pacientes do sistema de saúde privado. }\end{array}$ & $\begin{array}{l}\text { Estudo prospectivo } \\
\text { de prevalência }\end{array}$ \\
\hline $\begin{array}{c}\text { Souza et al. } \\
(2020)\end{array}$ & $\begin{array}{c}\text { Conhecimento de } \\
\text { enfermeiros sobre sepse } \\
\text { e choque séptico em um } \\
\text { hospital escola }\end{array}$ & $\begin{array}{l}\text { A maioria dos enfermeiros estudaram } \\
\text { sobre sepse durante a graduação, mas } \\
40,4 \% \text { referiram ter dificuldades no } \\
\text { cuidado ao paciente séptico, } 70,2 \% \\
\text { responderam erroneamente sobre a } \\
\text { atual definição de sepse, } 51 \% \text { ou } \\
\text { elencaram sinais não utilizados para }\end{array}$ & $\begin{array}{c}\text { Transversal } \\
\text { descritivo }\end{array}$ \\
\hline
\end{tabular}




\begin{tabular}{|c|c|c|c|}
\hline & & $\begin{array}{c}\text { identificação da sepse ou descreveram } \\
\text { apenas sinais da síndrome da resposta } \\
\text { inflamatória sistêmica. }\end{array}$ & \\
\hline $\begin{array}{c}\text { Massabani \& Silveira } \\
\text { (2021) }\end{array}$ & $\begin{array}{l}\text { Atuação do enfermeiro } \\
\text { no diagnóstico da sepse }\end{array}$ & $\begin{array}{c}\text { O conhecimento que o } \\
\text { enfermeiro possui } \\
\text { sobre a sepse junto com sua atuação } \\
\text { na } \\
\text { Sistematização da Assistência de } \\
\text { Enfermagem (SAE) } \\
\text { é o que vai garantir uma assistência de } \\
\text { qualidade, e assim } \\
\text { aumentar as chances de um bom } \\
\text { prognóstico para o paciente. }\end{array}$ & $\begin{array}{c}\text { Revisão } \\
\text { bibliográfica com } \\
\text { objetivo } \\
\text { exploratório }\end{array}$ \\
\hline $\begin{array}{l}\text { Santos et al. } \\
\qquad(2021)\end{array}$ & $\begin{array}{l}\text { Conhecimento do } \\
\text { enfermeiro sobre os } \\
\text { parâmetros de alerta da } \\
\text { sepse na triagem precoce } \\
\text { em terapia intensiva }\end{array}$ & $\begin{array}{l}\text { O enfermeiro que é conhecedor dos } \\
\text { parâmetros de alerta da sepse, bem } \\
\text { como dos protocolos institucionais e } \\
\text { do quadro clínico do paciente, tende a } \\
\text { atuar de maneira rápida no } \\
\text { reconhecimento e a melhorar o } \\
\text { prognóstico desse paciente. }\end{array}$ & Revisão integrativa \\
\hline
\end{tabular}

Fonte: Dados da pesquisa, 2021.

Mediante a leitura dos estudos, observou-se que a revisão foi comporta por 21 artigos. A maioria dos estudos foram publicados no ano de 2019 com 06 artigos, seguido dos anos 2018 com 05 artigos, 2020 com 03 artigos, 2021, 2017 com 02 e 2016 com 03 artigos. Em relação ao idioma 19 foram publicados em português e 02 em inglês. No que se refere ao continente de origem dos estudos 19 são da América do Sul, 01 artigo da América do Norte e 01 da Europa. No que concerne ao local onde ocorreram as pesquisas 11 ocorreram em unidades de terapia intensiva, 07 através de levantamento bibliográfico e 03 em enfermaria.

Desses 21 artigos 08 abordam com maior relevância sobre a equipe de enfermagem no atendimento ao paciente séptico e sua importância no reconhecimento precoce dessa complicação. Foram observados ainda 06 estudos evidenciando a necessidade de protocolos a fim de elevar a eficácia no atendimento de enfermagem após sua identificação e 07 apresentando a associação de ambos para melhoria da assistência na identificação da sepse.

\section{DISCUSSÃO}

Com base nos estudos analisados, verificou-se que a prevenção de infecções está diretamente ligada a assistência de enfermagem, e nesse panorama a equipe e o enfermeiro desempenham um papel fundamental no que diz respeito aos cuidados relacionados à sepse e as disfunções por ela causadas. Segundo Massabani \& Silveira (2021), diariamente as equipes de enfermagem atuam com pacientes críticos diagnosticados com sepse em unidades de terapia intensiva. Mediante a isso, o manejo à essa clínica envolve a necessidade do profissional obter o conhecimento necessário para reconhecer os sinais e sintomas ao surgirem, os identificando o mais brevemente possível, contribuindo para o diagnóstico precoce e um bom prognóstico ao indivíduo. 
Sobretudo, o enfermeiro possui importante relevância frente aos cuidados e intervenções. Seu papel no reconhecimento de alterações é imprescindível, uma vez que o diagnóstico e tratamento tardio diminuem as chances de sobrevida. Detentor de um olhar crítico sobre ela, agrega impactos positivos na sua identificação, monitorização e prevenção, sendo capazes de identificar a maioria das particularidades e características clínicas colaborando beneficamente para melhoria da atenção prestada (Branco et al., 2020).

Um estudo realizado no Paraná por Scheidt et al. (2018), destaca a importância da implementação de protocolos com o intuito de que o enfermeiro possa ser norteado quanto a sua atuação perante a alterações sugestivas à sepse. Outro estudo realizado em um hospital comunitário na Noruega observou após implementar ferramentas de triagem de sepse adequada para as enfermarias, que ocorreu um elevado número de identificação de casos de sepse de 6,7 para 84,2\% no hospital 1 e um aumento de 22,6\% para 45,2\% no hospital 2. Constatando que é imprescindível instituir sistemas padronizados por meio de protocolos, a fim de atenuar os índices de mortalidade e consequentemente diminuir a necessidade da assistência avançada em unidades de terapia intensiva (Torsvik et al., 2016).

Nos estudos analisados, identificou-se especialmente a necessidade da atenção dos enfermeiros voltada para identificação da sepse, não somente visando evitar diagnósticos tardios, mas sim para implementação de estratégias terapêuticas e de cuidados eficazes. Pedrosa, Oliveira \& Machado (2018), afirmam que quando há o reconhecimento em tempo hábil por meio da instituição de protocolos elaborados estrategicamente com embasamento científico, consequentemente aprimora a qualidade da assistência no controle da infecção e reabilitação do indivíduo séptico.

Dessa forma, estabelecer protocolos mais precisos, treinamentos com maior rigorosidade e conscientização, a fim de propiciar o melhor desempenho do profissional e a associação efetiva do quadro clínico do paciente com os sinais e sintomas sugestivos à sepse com maior eficácia, sucede em ações rápidas, seguras e direcionadas. A atuação precoce do enfermeiro atenua a incidência de disfunções generalizadas, obtendo ganhos em saúde, diminuindo impactos da doença e influenciando na melhoria da qualidade de vida do paciente séptico (Oliveira et al., 2019; Branco et al., 2020).

Nessa perspectiva, tendo em vista os benefícios da atuação, o reconhecimento prévio da sepse proporciona benefícios importantes quanto ao período de internação, por exemplo. Uma vez que quanto maior o intervalo de tempo a internação na UTI ocorrer, maiores as chances do paciente desenvolver uma infecção e quando esse período ultrapassa setenta e duas horas, a probabilidade do paciente evoluir com sepse é ainda maior. Ademais, no que se refere ainda ao período que um indivíduo permanece internado na unidade de terapia intensiva, Costa et al. (2019) diz que quanto mais tempo o paciente continuar na UTI, maior é a chance de favorecer também a disseminação de múltiplas infecções na unidade de terapia intensiva.

No que se refere aos fatores de risco relacionados a sepse em pacientes que se encontram em unidades de terapia intensiva, foi observado que o tempo de permanência hospitalar e os procedimentos invasivos como ventilação mecânica e cateterismo estão associados a um número elevado de óbitos por sepse na UTI. E a 
elevação do risco associa-se também a fatores variáveis de suma relevância para o seu desencadeamento, como as doenças de base, acessos endovenosos, sondagens vesicais e outros procedimentos que debilitam a proteção natural do organismo (Barros et al., 2016; Moura et al., 2017).

Essa circunstância é preocupante, visto que a sepse representa a principal causa de morte nas unidades de terapia intensiva não cardiológicas, especialmente no que se refere a disfunção múltipla de órgãos. Com isso, as intervenções a serem realizadas demandam cuidado, atenção e amplo conhecimento dos profissionais da enfermagem. Devendo esses obter preparo para atuação frente a essa clínica, a qual não recebendo a assistência adequada poderá ocasionar sérias complicações (Barros et al., 2016; Santos et al., 2021).

Considerando isso, as chances do paciente progredir para óbito são altas, e essa elevada mortalidade por sepse grave possui relação com a abordagem inadequada do agente etiológico. Com isso, o diagnóstico dessa complicação também é realizado por meio de achados clínicos, seguido do laboratorial. Logo, intervenções que visem a prevenção, reconhecimento precoce, tratamento eficaz, bem como a instituição de protocolos e práticas são providências que podem minimizar os índices de morbimortalidade e atenuar a exposição de pacientes a agentes causadores de sepse (Costa et al., 2019).

Nesse contexto, a Sistematização da Assistência de Enfermagem (SAE) é uma importante ferramenta utilizada pelo profissional enfermeiro na identificação de problemas, diagnóstico de enfermagem, estabelecimento de um planejamento de cuidados e sua implementação seguida de uma avaliação. Esses pontos sistematizam as ações da enfermagem viabilizando uma assistência com qualidade, possibilitando a construção de um plano de cuidados de acordo com as necessidades inerentes a cada um (Massabani \& Silveira, 2021).

Todavia, considerando a acelerada evolução da doença e a gravidade pertencente a ela, é crucial que os profissionais estejam preparados e atentos a predisposição de cada paciente e aos fatores de risco os quais se encontram expostos. Uma vez que o enfermeiro é fundamental no reconhecimento e avaliação precoce de manifestações clínicas que possam ocorrer nos pacientes. O profissional enfermeiro acompanha ainda o paciente em suas necessidades humanas básicas e propõe junto a equipe multiprofissional a elaboração e implementações assertivas da prevenção dessa patologia, no intuito de diminuir a morbimortalidade por sepse (Viana, Machado \& Souza, 2017; Silva \& Souza, 2018).

Em contrapartida, ainda são encontradas barreiras para atenuar a problemática, principalmente no quesito diagnóstico precoce e tratamento adequado. Nessa conjuntura, o paciente séptico deve ser avaliado de maneira holística pelo enfermeiro, o qual detém um papel imprescindível na identificação dessa complicação, devido ao seu contato constante beira leito. Com isso, é fundamental que obtenha treinamento adequado, comunicação e articulação eficaz entre si e com as demais áreas especializadas para evitar atrasos nas demandas do paciente (Kleinpell, 2017; Silva \& Souza, 2018; Zonta et al., 2018; Branco et al., 2020).

Uma vez que os atrasos acontecem e a comunicação sucede inadequadamente entre as equipes, pode ocorrer o comprometimento do diagnóstico e seu tratamento colaborando para a progressão da sepse. Favorecendo mutuamente as falhas e efeitos adversos que posteriormente poderão ocasionar malefícios, 
estender o tempo de internamento do paciente e consequentemente aumentar o consumo de recursos em saúde. Todavia, são pontos que podem ser evitados e conduzidos a um fim positivo (Silva \& Souza, 2018).

No entanto, o manejo ineficaz da sepse por vezes pode ainda estar associado ao déficit de conhecimento sobre a clínica do paciente, em detrimento de falhas na formação profissional. Essa situação demanda especialmente da equipe de enfermagem no que tange a identificação de disfunções orgânicas e das manifestações clínicas da sepse sobre a diferenciação das que apenas se assemelham a ela, porém são advindas de outros processos patológicos. Dessa forma, evidencia-se a importância da capacitação da equipe no que se refere ao manejo do paciente séptico, bem como maior envolvimento das Instituições no que diz respeito a treinamentos voltados para equipe (Oliveira et al., 2019; Alvim et al., 2020).

Para tanto, o enfermeiro enquanto líder da equipe de enfermagem precisa dispor de um conhecimento amplo, visando planejamento da assistência com competência e habilidade. Equivalente a um multiplicador de boas práticas dentro de sua equipe, é primordial estabelecer segurança quanto a tomada de decisões e coordenação. Incentivando de maneira oportuna com fundamento técnico-científico as intervenções de enfermagem no cuidado a pessoa acometida (Garrido et al., 2017; Fernandes et al., 2018).

Ressaltado a importância da equipe de enfermagem, é de grande valia que a implementação de ações de cunho educativo aos profissionais envolvidos corrobora diretamente na melhoria do nível de conhecimento, gestão e prática assistencial. A inserção de programas e projetos de educação continuada provindos das instituições em saúde podem acarretar em maior eficiência no atendimento, no diagnóstico precoce, tratamento propício e em avanços estratégicos no que concerne a redução de agravos associados à disfunções orgânicas (Goulart et al., 2019; Alvim et al., 2020).

Ademais, espera-se que esta revisão possa contribuir no que concerne ao destaque da importância do aperfeiçoamento das habilidades do enfermeiro mediante o paciente com sepse. Uma vez que o estudo enfatiza que o cuidado de enfermagem deve ser baseado em um fundamento técnico-científico, e sua assistência amparada por uma padronização e consequentemente uma linguagem eficaz entre a equipe. Influenciando beneficamente e proporcionando impactos positivos à assistência favorecendo a realização de intervenções mais ágeis e com segurança, além da prevenção com maior eficácia.

Diante disso, as limitações para elaboração deste estudo pautam-se na escassez de trabalhos relacionados a assistência direta da enfermagem, bem como a abordagem a temática utilizada de maneira ampla, contemplando as intervenções direcionadas. Dessa forma, é importante que mais estudos sejam realizados referente a questão do assunto, com o intuito de aprofundar o conhecimento disponível e otimizar as vertentes possibilitando o favorecimento da assistência de enfermagem ao paciente séptico.

\section{CONSIDERAÇÕES FINAIS}


Mediante a compilação dos argumentos apresentados foi possível evidenciar que o enfermeiro junto a sua equipe comporta um papel fundamental frente ao cuidado ao paciente crítico com sepse. Os estudos demonstram ainda a importância do papel destes na prevenção, diagnóstico e tratamento. Alinhando a necessidade de deter conhecimento sobre a doença, para que possa haver um monitoramento eficaz, com a finalidade de identificar os sinais iniciais da sepse, a fim de uma intervenção estratégica no menor tempo hábil possível.

Com isso, o enfermeiro por possuir maior contato e proximidade no atendimento ao paciente, ele deve estar apto para orientar e capacitar a sua equipe para a identificação dos primeiros sinais. Uma vez que evidências apontam que a eficiência na agilidade do atendimento ao paciente acometido por sepse está diretamente ligada ao diagnóstico precoce deste, fomentando assim que conhecer as características da sepse é fundamental.

Desse modo, podemos considerar que os objetivos deste estudo foram alcançados, uma vez que foram evidenciados impactos positivos e negativos mediante a assistência de enfermagem ao paciente séptico que se encontra na unidade de terapia intensiva. Demonstrando os benefícios e resultados que o conhecimento com afinco sobre essa disfunção orgânica associada a uma equipe capacitada e preparada proporcionam, assim como a elaboração e adesão a protocolos relacionados a identificação dessa disfunção influenciam em uma conduta precoce e assertiva.

\section{Conflitos de interesses}

Os autores declaram que não há conflitos de interesse. Todos os autores estão cientes da submissão do artigo.

\section{REFERÊNCIAS}

Alvim, A. L. S, Silvano, L. M, Ribas, R. T, Rocha, R. L. P. (2020). Conhecimento da equipe de enfermagem em relação aos sinais e sintomas da sepse. Revista Enfermagem em Foco. 11 (2), 133-138.

Barros, L. S., Maia, C. S. \& Monteiro, M. C (2016). Fatores de risco associados ao agravamento de sepse em pacientes em Unidade de Terapia Intensiva. 24 (4), 388-396.

Branco, M. J. C, Lucas, A. P. M, Marques, R. M. D, Sousa, P. P. (2020). O papel o enfermeiro perante o paciente com sepse. Revista Brasileira de Enfermagem. 73 (4), 1-8.

Costa, M. B. V, Ponte, K. M, Frota, A, K. C, Moreira, A. C. (2019). Características epidemiológicas de pacientes com sepse em unidade de terapia intensiva. Revista de Epidemiologia e Controle de Infecção. 9 (4), 310-315.

Fernandes, A. M., Sena, D. C., Soares, G. T., Carvalho, G. A., Nascimento, L. K., Pellense, M. C. (2018). Atuação da enfermagem na detecção precoce e tratamento da sepse na terapia intensiva. Revista Humano ser. 1 (1), 66-83. 
Garrido, F., Tieppo, L., Pereira, M. D., Freitas, R.., Freitas, W. M., Filipini, R.., Coelho, P. G., Fonseca, F. L., Fiorano. (2018). Ações do enfermeiro na identificação precoce de alterações sistêmicas causadas pela sepse grave. Revista ABCS Health Sciences. 42 (1), 15-20.

Gonçalves, J. R. (2019). Como escrever um artigo de revisão de literatura. Rev JRG de Estudos Acadêmicos. v. 2, n.5, p. 29-55.

Goulart, L. S., Júnior, M. A., Sarti, E. C. F, Sousa, Á. F. L., Ferreira, A. M., Frota, O. P. (2019). Are nurses updated on the proper management of patients with sepsis?. Escola Anna Nery. 23 (4), 1-6.

Kleinpell, R. (2017). Promoting early identification of sepsis in hospitalized patients with nurse-led protocols. Critical Care. 21 (10), 1-3.

Massabani, R. \& Silveira, G. C. (2021). Atuação do enfermeiro no diagnóstico da sepse. Revista Intersaúde. 1 (4), $59-65$.

Moura, J. M., Bertoli, E. S., Pereira, R. M., Frutuoso, I. S., Werneck, A. L., Contrin, L. M. (2017). Diagnóstico de sepse em pacientes após internação em unidade de terapia intensiva. Arq. Ciênc. Saúde. 21 (3), 55-60.

Moura, L. C., Pedreira, L. R.., Cruz, R. S., Moraes, A. C. (2019). Produção Científica Sobre Sepse, Terapia Intensiva e Enfermagem: Análise Bibliométrica do Período de 2003-2018. Revista Brasileira de Saúde Funcional. 8. (1), 119-130.

Ramalho Neto, J. M., Almeida, A. R., Silva, L. M., Viana, R. A., Nóbrega, M. M. (2019). Paciente grave com sepse: concepções e atitudes de enfermeiros intensivistas. Enfermagem Brasil. 18 (50), 650-657.

Oliveira S. C., Corrêa, B. T., Dodde, H. N., Pereira, G. L., Aguiar, B. G. (2019). O enfermeiro na detecção dos sinais e sintomas em pacientes na enfermaria. Fundam. Care. 11 (5), 1307-1311.

Pedrosa, K. A., Oliveira, S. A. \& Machado, R. C. (2018). Validação de protocolo assistencial ao paciente séptico na Unidade de Terapia Intensiva. Revista Brasileira de Enfermagem. 71 (3), 1172-80.

Santos, R. S., Salles, M., Matos, W., Navarros, A. A., Santos, A. J., Silva, I, A. (2021). Enfermagem: desafios e perspectivas para a integralidade do cuidado. Conbecimento do enfermeiro sobre os parâmetros de alerta da sepse na triagem precoce em terapia intensiva. (p. 105-118). Guarujá: Editora Científica.

Scheidt, S. N., Bordin, D., Aguiar, L. N., Tracz, E. C., Arcaro, G., Farago, P. V., Rocha, M. D. (2018). Implantação do protocolo de Manejo de sepse o pronto atendimento do hospital universitário regional dos campos gerais. Revista de Epidemiologia e Controle de Infecção. 8 (1), 54-64.

Silva, A. P. R. \& Souza, H. V. (2018). Sepse: importância da identificação precoce pela enfermagem. Revista PróUniversus. 9 (1), 47-100.

Souza, T. V., Melchior, L. M., Bezerra, F. S., Filha, F. S., Santos, O. P., Pereira, M. C., Félis, K. C., Filho, I. M. (2020). Conhecimento de enfermeiros sobre a sepse e choque séptico em um hospital escola. Journal Health NPEPS. 5 (1), 132-146.

Taniguchi, L. U., Azevedo, L, C., Bozza, F. A., Cavalcanti, A. B., Ferreira, E. M., Carrara, F. S., Sousa, J. L., Salomão, R.., Machado, F. R. (2019). Disponibilidade de recursos para tratamento da sepse no Brasil: uma amostra aleatória de instituições brasileiras. Revista Brasileira de Terapia Intensiva. 31 (2), 193-201

Torsvik, M. Gustad, L. T., Mehl, A., Bangstad, I. L., Vinje, J. K. D., Solligård, E. (2016). Early identification of sepsis in hospital inpatients by ward nurses increases 30-day survival. Critical Care. 20 (244), 2-9. 
Viana R. A., Machado F. R. \& Souza J. L. (2017). Sepse, um problema de saúde pública: a atuação e colaboração da enfermagem na rápida identificação e tratamento da doença. São Paulo: COREN-SP.

Zonta, F. N., Velasquez, P. G., Velasquez, L. G., Demetrio, L. S., Miranda, D., Silva, M. C. (2018). Características epidemiológicas e clínicas da sepse em um hospital público do Paraná. Revista de Epidemiologia e Controle de Infecção. 8 (3), 224-231.

\section{Contribuições dos autores}

Maressa Samai Pinheiro Silva Smith contribuiu com a coleta dos dados e a redação do manuscrito. Averlândio Wallysson Soares da Costa contribuiu com a análise e revisão crítica do manuscrito. 